\title{
GCU
}

Glasgow Caledonian

University

University for the Common Good

\section{Spatiotemporal and kinematic characteristics of gait initiation across a wide speed range}

Stansfield, Ben; Hawkins, Katy; Adams, Sarah; Church, Doireann

Published in:

Gait and Posture

DOI:

10.1016/j.gaitpost.2018.02.003

Publication date:

2018

Document Version

Author accepted manuscript

Link to publication in ResearchOnline

Citation for published version (Harvard):

Stansfield, B, Hawkins, K, Adams, S \& Church, D 2018, 'Spatiotemporal and kinematic characteristics of gait initiation across a wide speed range', Gait and Posture, vol. 61, pp. 331-338.

https://doi.org/10.1016/j.gaitpost.2018.02.003

\section{General rights}

Copyright and moral rights for the publications made accessible in the public portal are retained by the authors and/or other copyright owners and it is a condition of accessing publications that users recognise and abide by the legal requirements associated with these rights.

Take down policy

If you believe that this document breaches copyright please view our takedown policy at https://edshare.gcu.ac.uk/id/eprint/5179 for details

of how to contact us. 
Title page

Title: Spatiotemporal and kinematic characteristics of gait initiation across a wide speed range

Authors names and affiliations

Ben Stansfield, Katy Hawkins, Sarah Adams, Doireann Church

All authors are affiliated to: School of Health and Life Sciences, Glasgow Caledonian University, Cowcaddens Road, Glasgow, G4 OBA, UK.

Corresponding author

Ben Stansfield

Glasgow Caledonian University, Cowcaddens Road, Glasgow, G4 OBA, UK.

Email: ben.stansfield@gcu.ac.uk

Highlights (3-5 max 85 characters each)

- Gait initiation spatiotemporal and kinematic characteristics quantified.

- Outcomes developed across the full range of gait initiation speeds.

- Gait initiation characteristics change in a non-linear way with speed.

- Gender based differences in gait initiation are highlighted.

- Speed of performance must be taken into consideration when interpreting outcomes.

\section{Abstract}

Background 
Gait initiation can be performed at a range of speeds. Those with disability tend to use a slower speed compared to those without disability. In assessing the spatiotemporal and kinematic characteristics of gait initiation it is therefore important to consider the effects of speed on outcomes.

\section{Research Question}

What is the effect of speed of performance on spatiotemporal and kinematic characteristics of gait initiation?

\section{Methods}

Spatiotemporal and kinematic characteristics were measured across a wide range of speeds from very slow to very fast (normalised initiating leg (swing or SW limb) step speed 0.1-0.5) for 20 health adults (10 men/10 women, 22-44 years) using three-dimensional motion analysis of the first two steps of gait.

\section{Results}

Mixed linear modelling of 295 walking trials indicated differences between individuals, sexes and strong non-linear relationships between normalised initiating leg step speed and cadence and step lengths $\left(R^{2}>0.5\right)$. Particular characteristics of joint kinematics (maxima and minima for both initiating (SW) and contralateral limb (stance or ST limb)) demonstrated significant non-linear (squared, cubic and power law) changes with speed. Moderate to strong relationships were identified for sagittal plane pelvis, hip and knee kinematics as well as hip adduction $\left(0.3<R^{2}<0.7\right)$.

\section{Significance}

Gait initiation spatiotemporal and kinematic characteristics were quantified across the maximum range of speeds achievable, providing comprehensive characterisation of changes with speed. Significant, non-linear changes with speed were identified, suggesting different strategies are 
employed to modify speed at low and high speeds. The highlighted changes with speed illustrate the importance of taking speed into account when comparing outcomes between healthy adults and those with pathology.

\section{Keywords (up to 5)}

Gait initiation; speed; kinematics; spatiotemporal characteristics; normalisation; healthy adults

\section{Introduction}

Gait initiation requires the movement from a stable double leg stance to the dynamic state of walking. This action requires the coordinated movement of both lower limbs. Previous research has described typical movement patterns for a range of ages of healthy adults ${ }^{1-3}$ and for those with disability $^{4-7}$. These studies highlight the impact of lack of muscle control, or muscle weakness on the performance of gait initiation. It is therefore important to be able to quantify spatiotemporal characteristics and kinematics of this movement to assess the impact of disability. Typically studies report that participants are asked to perform the task at 'self-selected' speed ${ }^{1-5,8-10}$. However, if both healthy adults and those with disability are asked to perform gait initiation at 'self-selected' speed it is possible, even likely, that different speeds will be used. This is reflected in shorter duration and/or larger distances covered for typical gait initiation periods for healthy adults compared to those with disability (e.g. Parkinson's disease ${ }^{4,5,9,11}$ ). Whilst this results in apparent differences between the gait initiation characteristics, it may be that the differences in cadence, step length, timings and joint angles used are simply a result of choosing a different gait initiation speed. Indeed some forms of normalisation with respect to velocity of movement have been attempted previously ${ }^{4}$ reducing apparent 'differences' in gait initiation characteristics between groups. There are limited examples of the systematic exploration of changes in gait initiation spatiotemporal characteristics with speed. 
For example Breniere and Do ${ }^{12}$ instructed their participants to use 'slow', 'normal' and 'fast' speeds. However, individual's choices of what constitutes 'slow' and 'fast' will not be identical making grouping of results difficult. A systematic exploration of changes in gait initiation with speed allowing participants to initiate gait across a wide spectrum of speeds would remove the need for artificial self-selected speed bands. This would allow the exploration of true changes in gait initiation spatiotemporal characteristics (e.g. cadence, step length and phase timings) and joint kinematics (e.g. peak joint angles) with speed. This would be particularly helpful at the lower speeds of movement typical of those with severe pathology.

The aim of this study was therefore to characterise the relationship between speed of gait initiation and spatiotemporal characteristics and kinematics in healthy adults across a wide range of speeds of movement from the slowest to the fastest speeds. This will inform the interpretation of outcomes in studies involving groups of participants moving at different speeds. The hypothesis was that spatiotemporal characteristics and lower limb joint kinematics would change with speed of gait initiation.

\section{Methods}

Participants

Participants were recruited from staff and students at a UK higher education institution.

Institutional ethical approval was gained for the study and all participants gave written informed consent. Only participants between 18 and 60 years of age without lower limb impairment or neurological condition affecting the lower limb/walking function were recruited. An equal number of men and women were recruited. 


\section{Motion tracking}

The motion of participants was tracked (sixteen-camera motion analysis system, $120 \mathrm{~Hz}$ (Qualisys $A B$, Goteborg, Sweden)) using retro-reflective markers attached to the lower limbs (Table 1). Axes systems were defined as in Table 1. An initial static trial was used to establish the relationship between the cluster markers and their respective segment markers. This allowed the tracking of dynamic movements using the clusters. Joint angles were calculated as ordered sequences of rotations $(Z, X, Y)$ from the proximal to the distal coordinate systems at the respective joints.

Data collection protocol

Participants stood with feet a comfortable self-selected width apart at the start of a $6 \mathrm{~m}$ walk way. Following a verbal command the participants walked along the walk way stopping in a designated area at the end of the walk way. Participants were allowed several practice walks to become familiar with the procedure. The floor was carpeted. All participants walked with bare feet and wore shorts.

Initially participants walked at a self-selected normal speed for three walks. Then the participants were instructed to walk at incrementally slower speeds until they reached their slowest walking speed after 7 additional trials. Then the participants walked at their self-selected normal walking speed again for 3 trials before being asked to walk progressively faster to reach their fastest speed after 7 more trials. The aim of this protocol was to gain examples of walking across as wide a range of speeds as possible for each participant. This gave a total of 20 walks for each participant ranging in speed from their slowest to their fastest self-selected speed. 
Data analysis

In common with previous reports ${ }^{14}$ the leg that progressed first is referred to as the swing leg (SW) and the leg that progresses second is referred to as the stance leg (ST). Points of interest during the gait initiation were determined manually by examining graphical representation of specific marker displacement in conjunction with global movement of all markers attached to each participant. Consistent identification of timing points across all participants was checked by one of the authors (BS): ini - Initial movement of any of the markers; SWr1 - first heel raise of SW (timing of initial vertical movement in heel (HEE) marker); STr - stance leg heel raise; SWs - swing leg foot strike (timing of lowest point of HEE marker); SWr2 - second heel raise of SW; STs - stance leg foot strike. $100 \%$ of the gait initiation cycle was defined as from ini to STs.

\section{Spatiotemporal outcomes}

The following spatiotemporal outcomes were then calculated: Total time - from ini to STs; Preparation time - from ini to SWr1; SW Step time - from SWr1 to SWs; ST Step time - from STr to STs; SW Step length - R/LHEE from SWr1 to SWs; ST stride length - R/LHEE from STr to STs. From these measures the SW Step speed (length/time) and the SW Step cadence (1/SW Step time) were determined.

In an attempt to reduce the effect of differences in size of participants on the results a normalisation process was used ${ }^{15,16}$. This involved multiplying all variables by appropriate normalisation quantities as follows:

Normalised step/stride length $=$ step/stride length $\times(1 / \mathrm{BH})$

Normalised cadence $=$ cadence $\times(\mathrm{V}(\mathrm{BH} / \mathrm{g})$

Normalise speed $=$ speed $\times(1 /(\mathrm{V}(\mathrm{BH} \times \mathrm{g})))$

Normalised total/step time $=$ total/step time $\times(1 /(\mathrm{V}(\mathrm{BH} / \mathrm{g})))$ 
Where $\mathrm{BH}=$ body height, $\mathrm{g}=$ acceleration due to gravity $=9.81 \mathrm{~m} / \mathrm{s}^{2}$.

To examine the effects of speed on outcomes the normalised value of the SW step speed was used to represent the speed of the gait initiation. Mixed linear models were used to model the relationship between each of these outcomes and normalised SW step speed ${ }^{17}$. Models were adjusted for sex. Fixed and random effects were used at the participant (level2) and measurement (level 1) levels. The average growth curve for the sample was described by the fixed effects and the variation between participants by the random effects. A unique curve was therefore allowed for each participant based on deviation from the average curve. An unstructured covariance structure was used. Models were developed adding terms sequentially and comparing models for significant improvement (reduction of 3.84 in value of $-2 * \log L$ ikelihood $(-2 \mathrm{LnL}), \mathrm{p}<0.05)$. Maximum likelihood was used during model development for unbiased estimation of $-2 \mathrm{LnL}$, then restricted maximum likelihood for the final model to give unbiased estimates of coefficients. Model parameters and $95 \%$ $\mathrm{Cl}$ were calculated. Following the introduction of sex and allowing for an intercept, the models were then developed either using normalised SW step speed, its square and cube, or using a power law relationship (log transformed for model development) as appropriate. For timings a power law best fit was considered appropriate for those terms where very slow speed of gait initiation should result in very large outcomes.

\section{Kinematic outcomes}

To examine the effects of speed on joint angles all trials of all participants were grouped by the normalised SW step speed into 0.1 bands and the mean angle profiles of these bands plotted to gain a visual interpretation of outcomes. Normalised speed bands covering the range from 0.1 to 0.5 were used. From the graphical patterns of the joint angles specific features were identified that appeared to change with normalised speed. These outcomes (maxima and minima of joint angles) were determined. The relationship between these features and normalised SW step speed was investigated using mixed linear modelling as detailed above. 
Statistical analysis was performed in SPSS v23 (SPSS Inc, Chicago, IL). The values of $\mathrm{R}^{2}$ were calculated and used to categorise the fit of the models using a pragmatic approach: $<0.1$ very weak, 0.1-0.3 weak, 0.3-0.5 moderate, 0.5-0.7 strong, $>0.7$ very strong.

\section{Results}

Twenty participants (10 men/10 women) took part in this study ((mean $\pm S D)$ age $25.7 \pm 5.1$, range 22 44 years, height $1.72 \pm 0.11 \mathrm{~m}$, weight $69.4 \pm 14.6 \mathrm{~kg}$ ). Results in this study used normalised values. To convert data from these values to the original units the following should be applied for an average person of height $1.72 \mathrm{~m}$ :

step $/$ stride length $=$ normalised step/stride length $\times$ Height $=$ normalised length $\times 1.72$ (length, $\mathrm{m})$ cadence $=$ normalised cadence $\times(1 / \mathrm{V}($ Height $/ g)=$ normalised cadence $\times 2.39 \quad$ (cadence, steps $/ \mathrm{s})$ speed $=$ normalised speed $\times(V($ Height $\times g)))=$ normalised speed $\times 4.11 \quad($ speed, $\mathrm{m} / \mathrm{s})$ total $/$ step time $=$ normalised total $/$ step time $\times(V($ Height $/ g)))=$ normalised time $\times 0.42 \quad$ (time, $s)$ A total of 299 trials were successfully recorded (trials were excluded if marker tracking was poor, or participants did not perform the task correctly). No trials were recorded below a normalised SW step speed of 0.1, 29 between 0.1-0.2, 127 from 0.2-0.3, 106 from 0.3-0.4, 33 from 0.4-0.5 and 4 between 0.5 and 0.6. As so few trials were performed outside the normalised SW step speed range of 0.1 to 0.5 (equivalent to 0.41 to $2.05 \mathrm{~m} / \mathrm{s}$ for a person of height $1.72 \mathrm{~m}$ ) further analysis was restricted to this range of speeds and therefore examined 295 walking trials (mean 15 per person). Observation of the joint angles presented as means of the normalised SW step speed bands indicated distinct characteristics apparently changing with speed (Figure 1a-o). Following 
presentation of the relationship between the spatiotemporal parameters and normalised SW step speed (Figure 2), the kinematic outcomes selected for further analysis are presented graphically in Figure 3.

For all models intercept as a random parameter was significant (all $p<0.05)$, indicating that there were significant offsets between participants (Table 2). Sex was not a significant contributor to models for any of the spatiotemporal measures (Figure 2). However, it was for a number of the kinematic outcomes. In general women exhibited higher pelvic tilt, maximum pelvic obliquity (side up), maximum SW hip flexion, maximum SW hip adduction, maximum SW knee flexion 0-60\%, ST hip flexion at $100 \%$ and lower ST hip extension $50-80 \%$ than men (Table 2, Figure 3 ).

All spatiotemporal outcomes except preparation time demonstrated non-linear relationships with normalised SW step speed (Table 2, Figure 2). It appeared that within the lower speed ranges participants achieved higher speed by extending step/stride length rather than increasing cadence, whereas in contrast within the higher speed ranges that cadence was used to increase speed rather than extending step/stride length (Figures 1a-c). SW step cadence, SW step length, ST stride length demonstrated strong or very strong relationships with normalised SW step speed $\left(R^{2} \geq 0.526\right)$. Timings of gait initiation were either weakly or moderately related to normalised SW step speed except for preparation time which was only very weakly related $\left(R^{2}=0.036\right)$.

For the kinematic outcomes there were both linear and non-linear relationships with normalised SW step speed, ranging from strong (minimum pelvic tilt, SW maximum hip flexion, SW maximum hip adduction, ST maximum hip extension $50-80 \%$, ST hip flexion angle at $100 \%$ ) to very weak (ST maximum hip abduction $50-80 \%$, ST hip adduction at $100 \%$ ) and varying in strength between men and women. In general the men's models were better fits for the data (higher $\mathrm{R}^{2}$ ) than women's. 
In general the range of motion used at the joints was higher the higher the normalised SW step speed. However, for some variables at higher speeds, e.g. ST maximum knee flexion and ST maximum plantarflexion $60-80 \%$, there was a trend for a reduced change with increasing speed.

\section{Discussion}

The objective of this study was to characterise the spatiotemporal characteristics and joint kinematics of gait initiation across a wide range of speeds. Strong and very strong relationships between spatiotemporal characteristics of gait initiation and normalised SW step speed suggested clear changes in the performance of the task with changing speed (Table 2, Figure 2). Also graphical representation of joint kinematics grouped by normalised SW step speed (Figure 1) clearly demonstrated speed related changes (Figure 3). Sex was important in describing several kinematic characteristics (Table 2) and there were differences between individual participants (significant random parameter of intercept). Using mixed linear modelling it was possible to isolate these effects, highlighting that particular kinematic characteristics of the leading and trailing limbs demonstrated relationships with normalised SW step speed. These results reinforce the need to take speed of task execution into account when evaluating performance of gait initiation. The full range of speeds that might be chosen during gait initiation were studied, with the fastest speeds being 5 times the slowest speeds (normalised SW step speed 0.1-0.5). The data set therefore provides a comprehensive characterisation across the gait initiation speed range.

The use of force plates to characterise the anticipatory postural adjustment at the initiation of movement has been reported previously ${ }^{18-20}$ highlighting the importance of changes in the distribution of loading to the feet in allowing gait initiation. It is possible that these changes drive 
the changes in spatiotemporal and kinematic outcomes observed in the current study with speed.

Further investigation of these aspects of speed related changes in gait initiation is warranted along with muscular control mechanisms ${ }^{21}$.

The higher women's pelvic anterior tilt, SW/ST hip flexion and SW/ST knee flexion point to a more flexed pattern of movement to achieve the same speed as men. There were also observed differences in pelvic obliquity and hip adduction between sexes which are possibly related to women having relatively larger inter-hip joint distance compared to men requiring women to use higher angles to maintain the same width of gait.

Inter-participant differences in all outcomes were identified through the mixed linear model as random effects. This indicates that there were offsets in outcomes between individuals, suggesting differences in the way stepping is initiated. However, despite these inter-individual differences there were also significant speed related effects.

There were strong relationships between the normalised speed, cadence and step length characteristics of gait initiation, which appeared to be non-linear. It appeared that at very slow speeds that step length is used to increase speed, but that higher than a normalised SW step speed of 0.4 that increased cadence is predominantly used to increase speed (Figure 2a-c). Preparation time did not show a clear trend with speed. Relationships between specific aspects of the movement of the centre of pressure during anticipatory postural adjustment and gait initiation velocity have been previously demonstrated ${ }^{17}$, but no previous reports of changes in preparation time as determined in the current study with speed are available.

Joint kinematics followed similar patterns to those previously reported ${ }^{1,14}$. There were several moderate to strong relationships between joint angle characteristics and normalised SW step speed, suggesting complex modification of joint angles with speed. Although in general an increased speed was associated with an increase in joint range of motion there appeared to be some trends 
suggesting changing strategies for increasing speed across the speed range. For example the relationships of ST maximum knee flexion (Figure 30) and ST maximum plantarflexion $60-80 \%$ (Figure $3 q)$ suggest that at higher speeds these angles do not change with increasing speed. Similarly pelvic minimum tilt (Figure 3a) and SW hip maximum flexion (Figure 3d) show trends towards reducing changes with increasing speed at the higher speeds. However, SW knee maximum flexion $60-80 \%$ (Figure 3h) appears to show increasing changes with speed at higher speeds. Some of these changes appear to support the hypothesis that within lower speed ranges higher speeds are achieved by increasing step length and that within higher speed ranges higher speeds are achieved by increasing cadence. However, there is clearly a complex interplay of the SW and ST limbs and across the joints of the lower limb.

The current study quantifies gait initiation outcomes over a wider speed range than previously typically reported. Reports of gait initiation at slow speeds $\left(0.4-0.5 \mathrm{~m} / \mathrm{s}^{14,22-23}\right)$ have been made. However, there appear to be only small studies exploring changes across wide speed ranges ${ }^{12,22}$. These studies either did not include the slower or faster speeds of gait initiation $(1.01-1.79 \mathrm{~m} / \mathrm{s}$ second step of gait initiation $\left.{ }^{12}, 0.4-1.3 \mathrm{~m} / \mathrm{s}^{22}\right)$. The current study's normalised speed range of $0.1-0.5$ was equivalent to a speed range of $0.41-2.06 \mathrm{~m} / \mathrm{s}$ (for a person of height $1.72 \mathrm{~m}$ ), wider than these previous reports. Typically studies only report outcomes at self-selected speed ${ }^{2,5,14,23-25}$. Outcomes such as step timings $s^{1,4,9,14}$, step lengths ${ }^{2-5,9,14,22}$ and cadence ${ }^{26}$ have been previously reported, but only at isolated speeds and not in relation to changes with speed. Due to variation in the definition of the characteristics of gait initiation it is difficult to directly compare the reported values with those reported here. However, in general there is agreement that at higher speeds of progression, step lengths are longer and timings quicker for the first steps of gait. 


\section{Conclusion}

Spatiotemporal and kinematic characteristics of gait initiation were quantified across the full range of gait initiation speeds from the slowest to fastest walking pace that participants could reasonably adopt. No significant differences between sexes in spatiotemporal outcomes were observed, but there were differences in joint angles between men and women. Significant relationships with speed for both spatiotemporal characteristics and joint kinematics of the lower limbs were observed. Of particular note was a non-linear relationship between gait initiation speed and cadence suggesting a change in strategy to increase speed from slow to fast speeds. Joint kinematic outcomes illustrate that this was achieved using a combination of joints across both limbs. These findings highlight the need to take speed of gait initiation into consideration when comparing those with disease or pathology with healthy control subjects.

\section{Acknowledgements}

The authors would like to thank all participants in the study. This research did not receive any specific grant from funding agencies in the public, commercial, or not-for-profit sectors.

\section{Conflict of Interest}

The authors have no conflict of interest in regards to this work.

\section{References}

1. Nissan M, Whittle MW, Initiation of gait in normal subjects: a preliminary study. J Biomed Eng 1990; 12: 165-171. 
2. Muir BC, Rietdyk S, Haddad JM, Gait initiation: The first four steps in adults aged 20-25, 65-79, and 80-91 years. Gait and Posture 2014; 39: 490-494.

3. Lu C, Amundsen Huffmaster SL, Harvey JC, MacKinnon CD, Anticipatory postural adjustment patterns during gait initiation across the adult lifespan. Gait and Posture 2017; 57:182-187.

4. Halliday SE, Winter DA, Frank JS, Patla AE, Prince F, The initiation of gait in young, elderly, and Parkinson's disease subjects. Gait and Posture 1998; 8:8-14.

5. Fernandez KM, Roemmich RT, Stegemoller EL, Amano S, Thompson A, Okun MS, Hass CJ, Gait initiation impairments in both essential tremor and Parkinson's disease. Gait and Posture 2013; 39: 956-961.

6. Chang HA, Chuang TY, Lee SJ Liao SF, Shih YH, Cheng H, Temporal differences in relative phasing of gait initiation and first step length in patients with cervical and lumbosacral spinal cord injuries. Spinal Cord 2004; 42:281-289.

7. Rajachandrakumar R, Fraser JE, Schinkel-Ivy A, Inness EL, Biasin L, Brunton K, Mcllroy WE, Mansfield A, Atypical anticipatory postural adjustment during gait initiation among individuals with sub-acute stroke. Gait and Posture 2017; 52:325-331.

8. Hartley EM, Hoch MC, McKeon PO, Reliability and responsiveness of gait initiation profiles in those with chronic ankle instability. Gait and Posture 2016; 49:86-89.

9. Roemmich RT, Nocera JR, Vallabhajosula S, Amano S, Naugle KM, Stegemoller EL, Hass CJ, Spatiotemporal variability during gait initiation in Parkinson's disease. Gait and Posture 2012; 36:340-343.

10. Dessery Y, Barbier F, Gillet C, Corbeil P, Does lower limb preference influence gait initiation? Gait and Posture 2011; 33:550-555. 
11. Okada Y, Fukumoto T, Takatori K, Nagino K, Hiraoka K, Abnormalities of the first three steps of gait initiation in patient with Parkinson's disease with freezing of gait. Parkinson's Disease 2011; 202937:1-8.

12. Breniere $\mathrm{Y}$, Do MC, When and how does steady state gait movement induced from upright posture begin? Journal of Biomechanics 1986; 19(12): 1035-40

13. Bell AL, Pedersen DR, Brand RA(1990) A Comparison of the Accuracy of Several hip Center Location Prediction Methods. J Biomech. 23, 617-621.

14. Mann RA, Hagy JL, White V, Liddell D, The initiation of gait. The Journal of Bone and Joint Surgery $1979 ; 61-A(2): 232-239$.

15. Stansfield BW, Hazlewood ME, Hillman SJ, Lawson AM, Loudon IR, Mann AM, Robb JE, Normalisation of gait data in children. Gait and Posture 2003; 17:81-87.

16. Hof AL. Scaling gait data to body size. Gait Posture 1996; 4:222-3.

17. Keene DJ, Moe-Nilssen R, Lamb SE, The application of multilevel modelling to account for the influence of walking speed in gait analysis. Gait and Posture 2016; 43:216-219.

18. Leppers $\mathrm{R}$, Breniere $\mathrm{Y}$, The role of anticipatory postural adjustments and gravity in gait initiation. Experimental Brain Research 1995; 107:118-24.

19. Vrieling AH, van Keeken HG, Schoppen T, Otten E, Halbertsma JPK, Hof AL, Postema K, Gait initiation in lower limb amputees. Gait and Posture 2008;27:423-430.

20. Sousa ASP, Silva A, Santos R, Ankle anticipatory postural adjustment during gait initiation in healthy and post-stroke subjects. Clinical Biomechanics 2015; 30(9):960-5.

21. Creena $P$, Frigo $C, A$ motor programme for the initiation of forward-oriented movements in humans. Journal of Physiology 1991; 437:635-653. 
22. Dibble LE, Nicholson DE, Shultz B, MacWilliams BA, Marcus RI, Moncur C, Sensory cueing effects on maximal speed gait initiation in persons with Parkinson's disease and healthy elders. Gait and Posture 2004; 19:215-225.

23. Park S, Choi H, Ryu K, Kim S, Kim Y, Kinematics, kinetics and muscle activities of the lower extremity during the first four steps from gait initiation to the steady-state walking. Journal of Mechanical Science and Technology 2009; 23:204-211.

24. Elble RJ, Moody C, Leffler K, Sinha R, The initiation of normal walking. Movement Disorders 1994;9(2):139-146.

25. Delval A, Krystkowiak P, Blatt J-L, Labyt E, Bourriez J-L, Dujardin K, Destee A, Dermbure P, Defebvre L, A biomechanical study of gait initiation in Huntington's disease. Gait and Posture 2007; 25:279-288.

26. Gormley JP, Barr DA, Bell AJ, Ravey J, Mollan RAB, Examination of the duration of gait initiation by use of an electrogoniometer. Gait and Posture 1993; 1:85-91. 
Figure 1. Joint angles of both legs from initiation of movement (0\%) to ST heel strike (100\%). Mean curves are presented of all trials walked within normalised speed bands: Lightest 0.1-0.2, then 0.20.3, 0.3-0.4 and darkest 0.4-0.5. Specific features characterised are highlighted with dashed lines/arrows. a) SW pelvic tilt (posterior tilt +ve) b) SW pelvic obliquity (side down +ve) c) SW pelvic rotation (side forward +ve) d) SW hip flexion/extension (flexion +ve) e) SW hip adduction/abduction (adduction +ve) f) SW hip internal/external rotation (internal +ve) g) SW knee extension/flexion (extension +ve) h) SW ankle dorsiflexion/plantarflexion (dorsiflexion +ve) i) SW foot progression angle (external rotation +ve). j)-o) contralateral side (ST) for equivalent of d)-i).

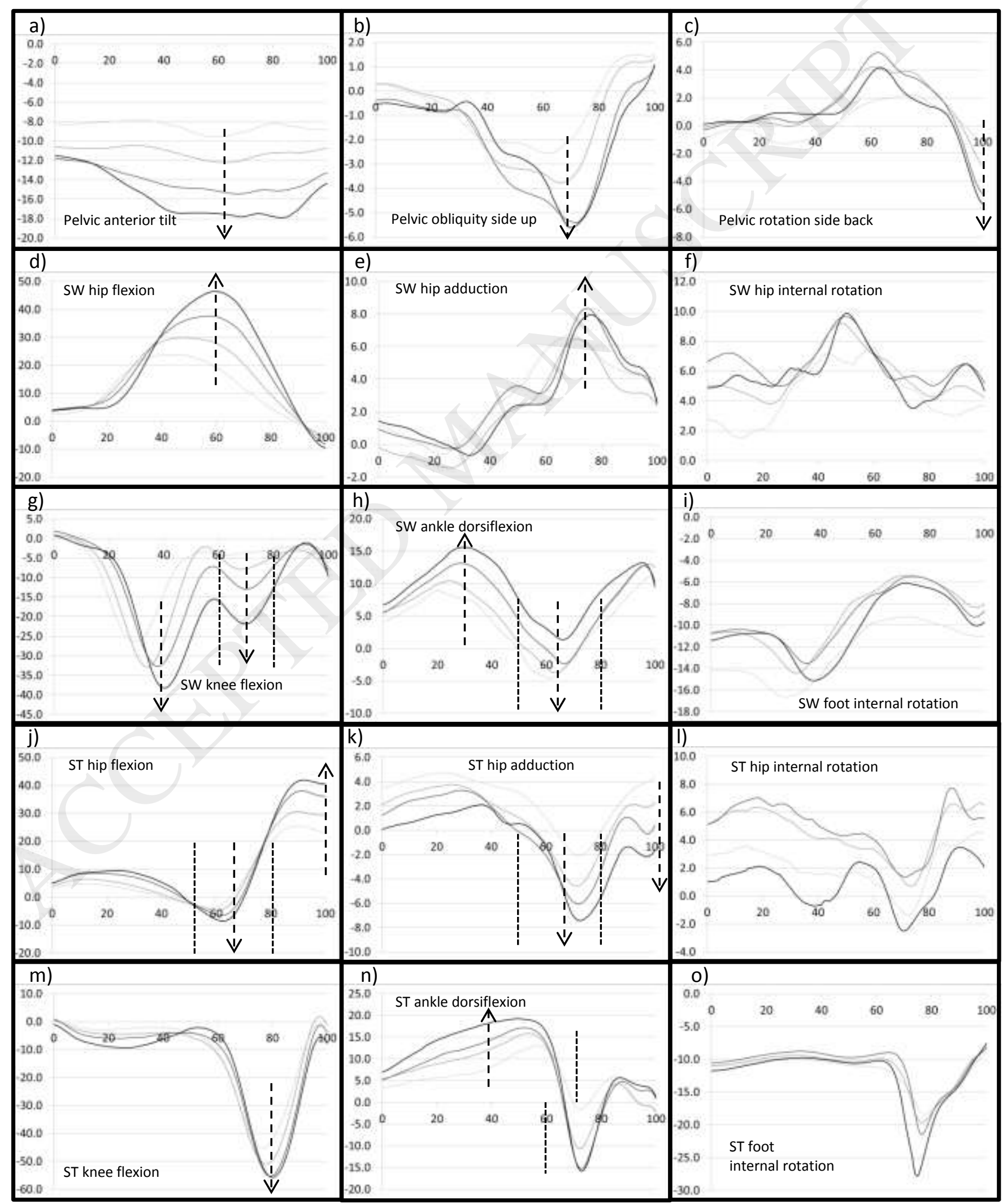


Figure 2 Best fit models (no sex difference) for normalised spatiotemporal outcomes against normalised SW step speed (refer to Table 3). Results for men are indicated with open squares and women with solid circles. All outcomes for all participants are illustrated. $R^{2}$ values are given.

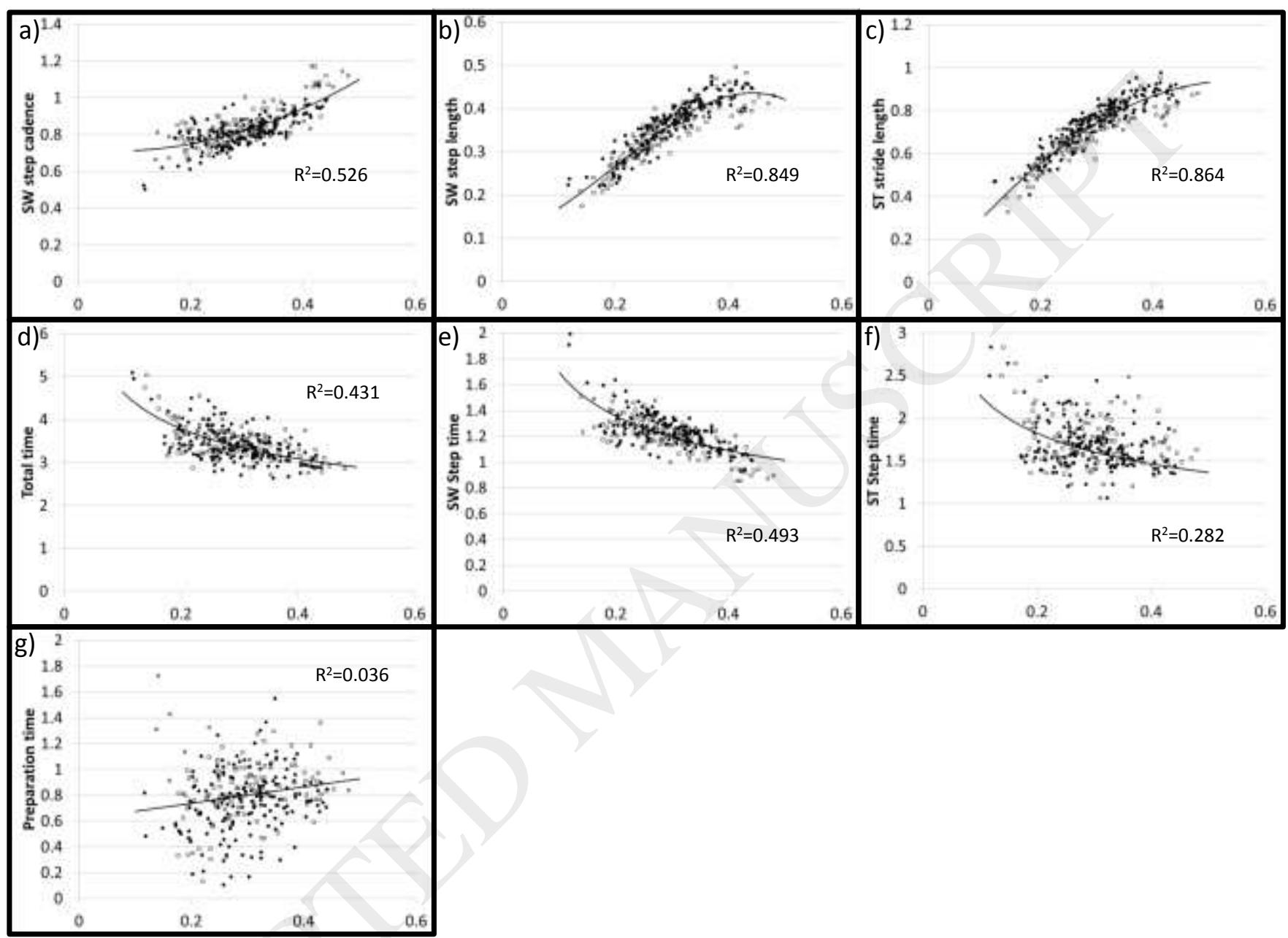

Figure 3 Best fit models (Table 3 ) for specific features of joint angles (degrees) against normalised SW step speed. $\min =$ minimum, $\max =$ maximum, percentages refer to the activity cycle. Results for men are illustrated with open squares (dashed line) and for women with closed circles (continuous line). Where sex was not a significant predictor in the model a single best fit solid line is given. All outcomes for all participants are illustrated. $R^{2}$ values are given. 


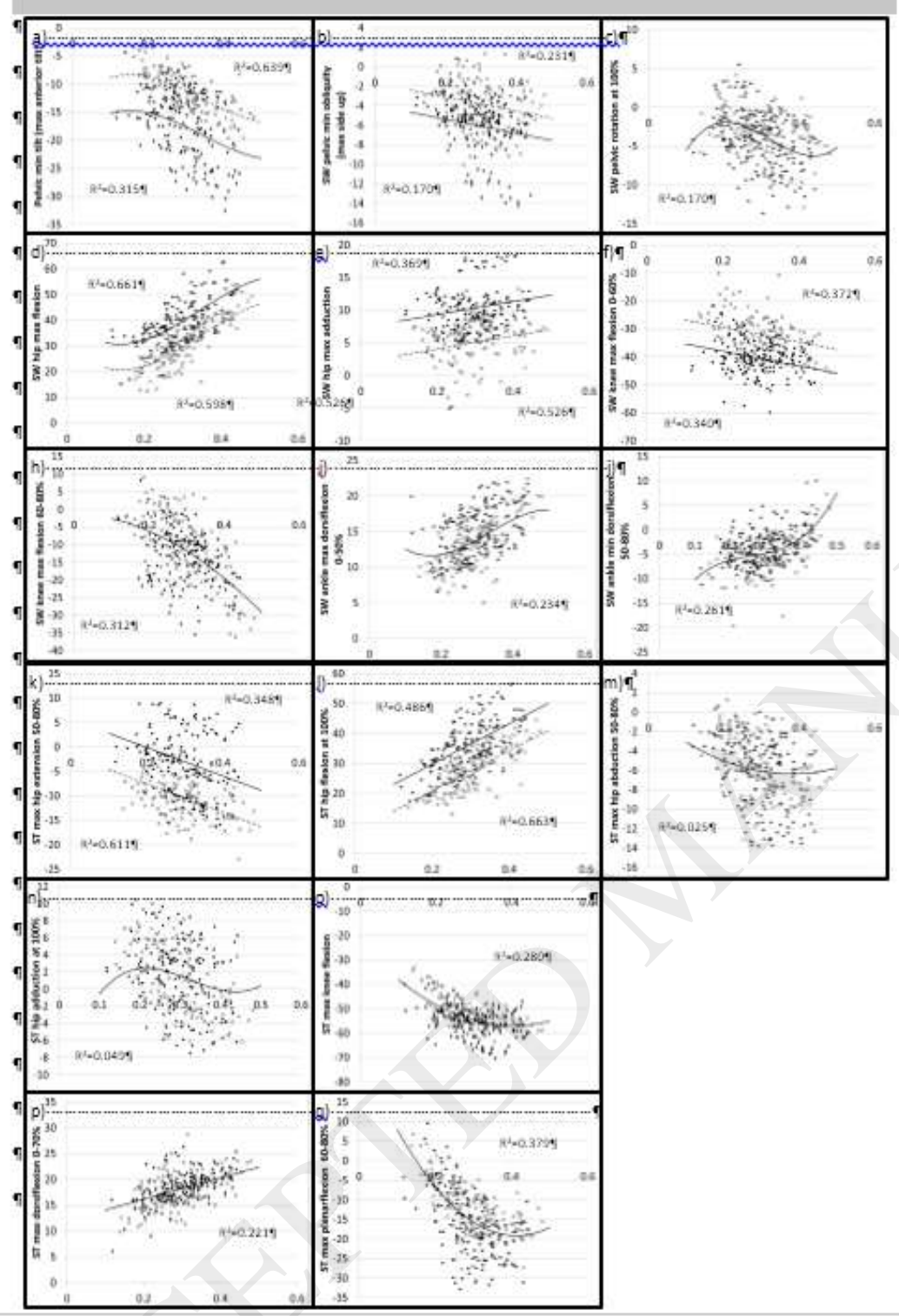

Table 1 Motion analysis marker locations and axes system definitions.

\section{Marker locations and definitions}

\begin{tabular}{|l|l|}
\hline Name & Location (Markers all either $19 \mathrm{~mm}$ or $\mathbf{1 6} \mathrm{mm}$ (ankle and foot) spherical) \\
\hline RASIS, LASIS & Anterior superior iliac spine \\
\hline
\end{tabular}




\begin{tabular}{|c|c|c|c|}
\hline \multicolumn{2}{|l|}{ RPSIS, LPSIS } & \multicolumn{2}{|l|}{ Posterior superior iliac spine } \\
\hline \multicolumn{2}{|l|}{ RGTR, LGTR } & \multicolumn{2}{|l|}{ Lateral to most prominent greater trochanter } \\
\hline \multicolumn{2}{|c|}{ RTHI1-4, LTHI1-4 } & \multicolumn{2}{|l|}{ Thigh cluster } \\
\hline \multicolumn{2}{|l|}{ RLKNE, LLKNE } & \multicolumn{2}{|l|}{ Lateral knee, $1 \mathrm{~cm}$ proximal to joint line at mid a-p knee } \\
\hline \multicolumn{2}{|c|}{ RMKNE, LMKNE } & \multicolumn{2}{|l|}{ Medial knee. Same height as RLKNE/LLKNE mid a-p knee } \\
\hline \multicolumn{2}{|c|}{ RSHA1-4, LSHA1-4 } & \multicolumn{2}{|l|}{ Shank clusters } \\
\hline \multicolumn{2}{|c|}{ RLANK, LLANK } & \multicolumn{2}{|l|}{ Lateral ankle, most prominent lateral malleolus } \\
\hline \multicolumn{2}{|c|}{ RMANK, LMANK } & \multicolumn{2}{|l|}{ Medial ankle, most prominent medial malleolus } \\
\hline \multicolumn{2}{|l|}{ RHEE, LHEE } & \multicolumn{2}{|l|}{ Mid line of heel at same level as mid-point of RMET1-5, LMET1-5 } \\
\hline RMET1,5, LMI & $T 1,5$ & Dorsal to metatarsal distal head & \\
\hline \multicolumn{4}{|c|}{ Axes system definitions } \\
\hline Lab axes & Origin & Axes & $\begin{array}{l}\text { Tracking } \\
\text { markers }\end{array}$ \\
\hline $\begin{array}{l}\text { Laboratory } \\
\text { axes }\end{array}$ & $\begin{array}{l}\text { Laboratory } \\
\text { origin }\end{array}$ & $\begin{array}{l}X=\text { direction of progression } \\
Y=\text { vertically upwards } \\
Z=\text { to right }\end{array}$ & $\begin{array}{l}\text { Qualisys } \\
\text { system }\end{array}$ \\
\hline Pelvis & $\begin{array}{l}\text { Midpoint of } \\
\text { ASIS (midASIS) }\end{array}$ & $\begin{array}{l}X=\text { from midPSIS to midASIS anteriorly } \\
Y=\text { perpendicular to both } X \text { and line from RASIS to LASIS } \\
\text { pointing superiorly } \\
Z=\text { perpendicular to both } X \text { and } Y \text { pointing to right }\end{array}$ & $\begin{array}{l}\text { RASIS, } \\
\text { LASIS, } \\
\text { RPSIS, } \\
\text { LPSIS }\end{array}$ \\
\hline Femur & Hip centre** & $\begin{array}{l}\text { interASIS = distance from RASIS to LASIS } \\
\text { KNEE = mid-point of MKNE and LKNE } \\
X=\text { perpendicular to } Y \text { and LKNE-MKNE pointing anteriorly. } \\
Y=\text { from KNEE to HIP proximally } \\
Z=\text { perpendicular to } X \text { and } Y \text { pointing to right. }\end{array}$ & $\begin{array}{l}\text { THI1, } \\
\text { THI2, } \\
\text { THI3, } \\
\text { THI4 }\end{array}$ \\
\hline Shank & KNEE & $\begin{array}{l}\text { ANK = mid-point of LANK and MANK } \\
X=\text { perpendicular to } Y \text { and LKNE-KNEE pointing anteriorly } \\
Y=\text { from ANK to KNEE proximally. } \\
Z=\text { perpendicular to } X \text { and } Y \text { pointing to right }\end{array}$ & $\begin{array}{l}\text { SHA1, } \\
\text { SHA2, } \\
\text { SHA3, } \\
\text { SHA4 }\end{array}$ \\
\hline Foot & $\begin{array}{l}\text { Vertical } \\
\text { projection of } \\
\text { HEE to level of } \\
\text { midMET }\end{array}$ & $\begin{array}{l}\text { midMET = mid-point of MET1, MET5 } \\
X=\text { from origin to midMET anteriorly } \\
Y=\text { perpendicular to } X \text { and horizontal projection (during static } \\
\text { trial) of line joining MET1-MET5 } \\
Z=\text { perpendicular to } X \text { and } Y \text { pointing right. }\end{array}$ & $\begin{array}{l}\text { MET1, } \\
\text { MET5, } \\
\text { HEE }\end{array}$ \\
\hline $\begin{array}{l}\text { Foot } \\
\text { progression } \\
\text { angle }\end{array}$ & & $\begin{array}{l}\text { The angle between the laboratory } X \text { axis and the line joining } \\
\text { HEE-midMET projected vertically onto the floor. }\end{array}$ & \\
\hline
\end{tabular}

**Offset from midASIS in Pelvis axes system: $\mathrm{X}=-0.19 *$ interASIS-0.5*Marker Diameter; $\mathrm{Y}=-$ $0.30 *$ interASIS; $Z=0.36 *$ interASIS $^{13}$.

Table 2 Mixed linear model results, both fixed and random parameters for all outcomes. For all outcomes the developed model is given with fixed and random parameters for significant terms included with $95 \%$ confidence intervals of the parameters. Coefficients which were not statistically significant are retained in the model where their sequential inclusion improved the level of fit. For 
the power law relationships, coefficients $a$ and $b$ are given for $y=a x^{b} . R^{2}$ calculated for fixed effects model either for both sexes together if sex was not significant in the model or separately if sex made a significant contribution.

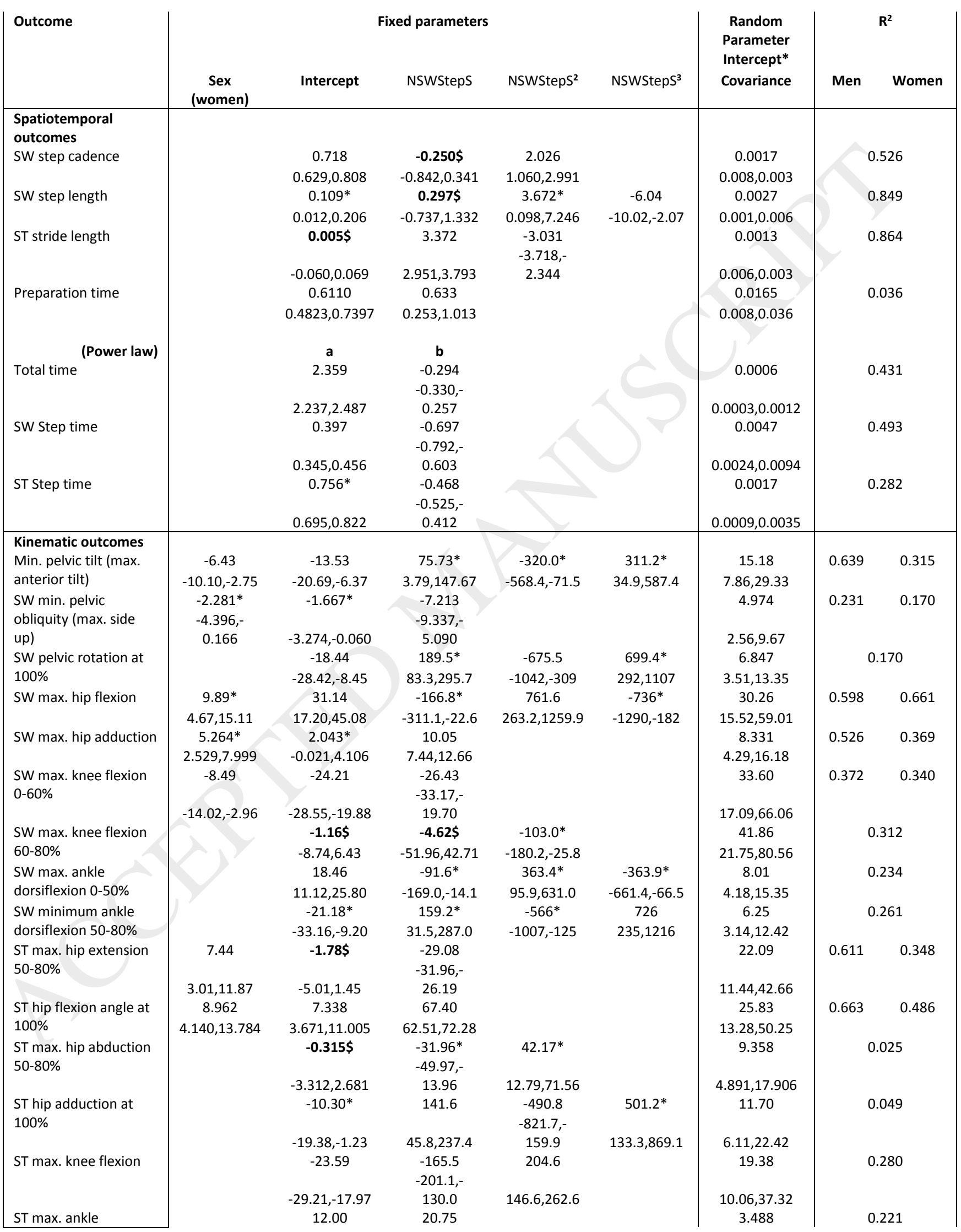


dorsiflexion 0-70\%

ST max. ankle

plantarflexion $60-80 \%$

$\begin{array}{ccc}10.60,13.40 & 17.08,24.43 & \\ 28.11 & -229.3 & 278.0 \\ & -277.7,- & \\ 20.63,35.59 & 180.9 & 199.0,356.9\end{array}$

$1.751,6.945$

22.76

0.379

$11.69,44.31$

NSWStepS $=$ normalised SW step speed. All coefficients significant at $p<0.001$ except $* 0.05>p \geq 0.001$, $\$ p>0.05$. Min. $=$ minimum, $\max .=$ maximum. Random intercepts by participant all $0.05>p \geq 0.001$. 